\title{
Optimization of PID Controller Parameters based on Genetic Algorithm for non-linear Electromechanical Actuator
}

\author{
Shayma'a A. Mahdi \\ College of Engineering, University of Baghdad.
}

\begin{abstract}
The servo motors system have been widely used in industrial robotics and electronics due to its excellent speed control characteristics even though its maintenance costs are higher than the induction motor. In this paper, the genetic algorithm were used for non-linear electromechanical actuator to determine the optimal parameters of the PID controller to improve the transient response of the system. The designed parameters which were optimized are rise time, peak time, settling time and maximum overshoot. The Simulation results show that the genetic algorithm is a fast and flexible tuning method to determine the optimal parameters of PID controller for wide range of requirements to achieve satisfied performance for stable system. Genetic Algorithm applied in PID controller improves transient response, the average percent overshoot reduction about $80 \%$ compared to the conventional methods such as optimized fuzzy supervisory PID, while keep the delay time, rise time and peak time almost unchanged and improves the settling time
\end{abstract}

Keywords: Electromechanical Actuator, Genetic Algorithm, PID controller, Optimization.

\section{1-INTRODUCTION}

The use of electromechanical actuation is becoming increasingly popular in the aerospace industry as more importance is placed on maintainability. Electromechanical actuators (EMAS) are being used in the actuation of flight critical control surfaces and in thrust vector control [1]. Electrical motor servo systems are indispensable in modern industries. Servo motors are used in a variety of applications in industrial electronics and robotics that includes precision positioning as well as speed control [2]. Servomotors use feedback controller to control the speed or the position, or both. The basic continuous feedback controller is PID controller which possesses good performance. However is adaptive enough only with flexible tuning. Although many advanced control techniques such as selftuning control, model reference adaptive control, sliding mode control and fuzzy control have been proposed to improve system performances, the conventional PI/PID controllers are still dominant in majority of real-world servo systems [1].To implement a PID controller the proportional gain $K P$, the integral gain $K I$ and the derivative gain $K D$ must be determined carefully. Many approaches have been developed to determine PID controller parameters for single input single output (SISO) systems.

\section{2-MATHEMATICAL SYSTEM MODEL}

\section{A-Linear model}

Consider a DC servo motor as shown in figure(1). A simple mathematical relationship between the shaft angular position and voltage input to the DC motor may be derived from physical laws. In the point of control system, DC servo motor can be considered as SISO plant [3]. Therefore, complications related to multi-input system are discarded. DC servo motors have the field coil in parallel with the armature. The current in the field coil and the armature are independent of one another. As a result, these motors have excellent speed and position control [4]

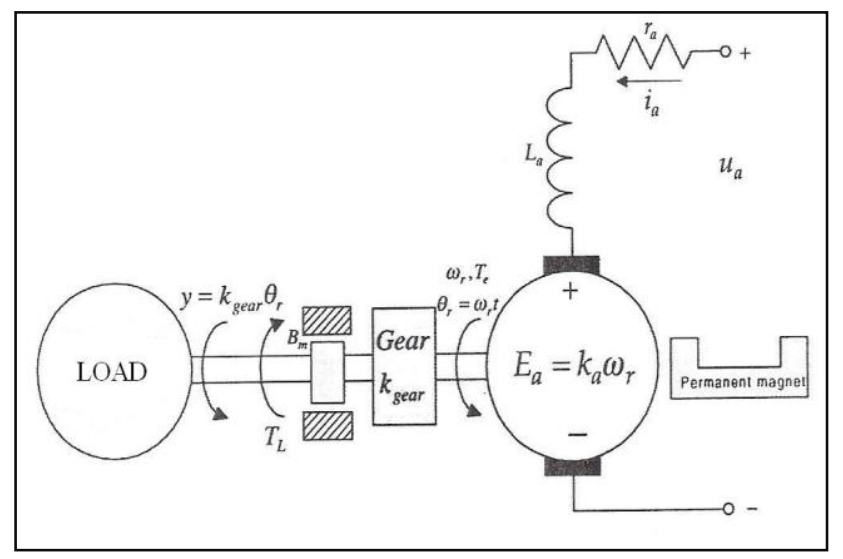

Fig. 1 Schematic diagram of the DC motor.

To find the transfer function for the block diagram of the open and closed loop system a differential equation used to describe the system dynamic. In the begging

Kirchhoff's voltage is use to map the armature circuitry dynamic of the motor.

$$
\frac{d i_{a}}{d t}=-\frac{r_{a}}{L_{a}} i_{a}-\frac{k_{a}}{L_{a}} \omega_{r}+\frac{1}{L_{a}} u_{a}
$$

Then using Newton's 2nd law

$\sum \bar{T}=J \bar{\alpha}=J \frac{d \bar{\omega}}{d t}$

The electromagnetic torque developed by the permanentmagnet DC motor

$T_{a}=k_{a} i_{a}$ 
The viscous friction torque

$T_{\text {viscous }}=B_{m} \omega_{r}$

The load torque is denoted as $\mathrm{T}_{\mathrm{L}}$. Use the Newton's second law, we have

$$
\begin{aligned}
& \frac{d \omega_{r}}{d t}=\frac{1}{J}\left(T_{a}-T_{\text {viscous }}-T_{L}\right)= \\
& \frac{1}{J}\left(k_{a} i_{a}-B_{m} \omega_{r}-T_{L}\right)
\end{aligned}
$$

The dynamics of the rotor angular displacement

$$
\frac{d \theta_{r}}{d t}=\omega_{r}
$$

To find the transfer function, the derived three first-order differential equation

$$
\begin{aligned}
& \frac{d i_{a}}{d t}=-\frac{r_{a}}{L_{a}} i_{a}-\frac{k_{a}}{L_{a}} \omega_{r}+\frac{1}{L_{a}} \\
& \frac{d \omega_{r}}{d t}=\frac{1}{J}\left(k_{a} i_{a}-B_{m} \omega_{r}-T_{L}\right)
\end{aligned}
$$

and

$$
\frac{d \theta_{r}}{d t}=\omega_{r}
$$

Using the Laplace operator

$$
s=\frac{d}{d t}
$$

$$
\left.s+\frac{r_{a}}{L_{a}}\right) i_{a}(s)=-\frac{k_{a}}{L_{a}} \omega_{r}(s)+\frac{1}{L_{a}} u_{a}(s)
$$

$$
\begin{aligned}
& \left(s+\frac{B_{m}}{J}\right) \omega_{r}(s)=\frac{1}{J} k_{a} i_{a}(s)- \\
& \frac{1}{J} T_{L}(s)
\end{aligned}
$$

$$
s \theta_{r}(s)=\omega_{r}(s)
$$

A simple model that could describe an actuator 's dynamics is a linear second - order system with demping ratio zeta $(\zeta)$ and natural frequency omega $\left(\boldsymbol{\omega}_{\mathbf{n}}\right)$. The transfer function of a second - order system is given below, where $\left(\omega_{r}\right)$ is the output and $\left(u_{\alpha}\right)$ is the input[5].
$\omega_{r}(\mathrm{~s})=\mathrm{G}(\mathrm{s}) \cdot u_{a}(\mathrm{~s})=\left(\omega_{\mathrm{n}}^{2} / \mathrm{s}\left(\mathrm{s}^{2}+2 \zeta \omega_{\mathrm{n}} \mathrm{s}+\omega_{\mathrm{n}}^{2}\right)\right)$ (14)

\section{B- Nonlinear Model}

The nonlinear model equations [6]:

$\mathrm{R}(\mathrm{s})=u_{a}(\mathrm{~s})-\omega_{r}(\mathrm{~s}) \operatorname{Lim}$
$\mathrm{Y}(\mathrm{s})=\mathrm{R}(\mathrm{s})[\mathrm{Kp}+\mathrm{Ki} / \mathrm{s}+\mathrm{s} \mathrm{Kd}]$

$\mathrm{s}^{2} \omega_{r}(\mathrm{~s})=\omega_{\mathrm{n}}^{2}\left[\mathrm{Y}(\mathrm{s})-\mathrm{RATEFB}-S \omega_{r}(s) \frac{2 \zeta}{\omega \mathrm{n}}\right]-$

HM

(17)

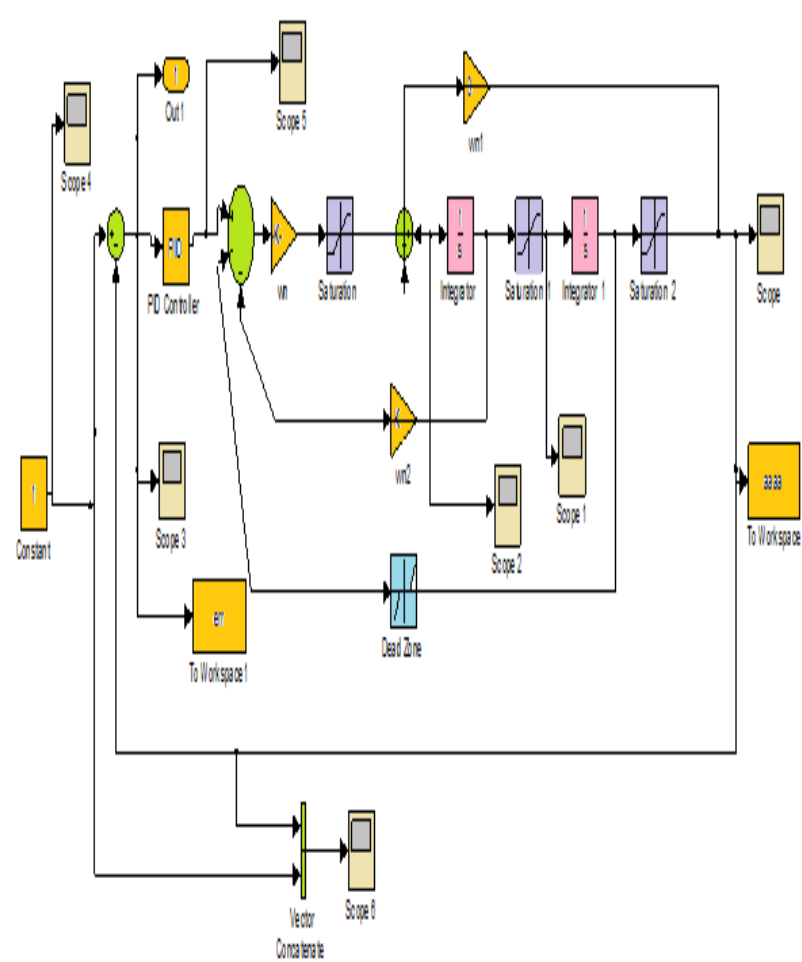

Fig.2 Block diagram of non-linear electromechanical servo motor to adjust PID parameters via GA.

\section{3- GENETIC ALGORITHIMS (GAs)}

Genetic algorithms are automated method for solving problems. GAs operate on a population of potential solutions applying the principle of survival of the fittest to produce better and better approximations to a solution. At each generation, a new set of approximations is created by the process of selecting individuals according to their level of fitness in the problem domain and breeding them together using operators borrowed from natural genetics. This process leads to the evolution of populations of individuals that are better suited to their environment than the individuals that they were created from, just as in natural adaptation as shown in figure (3) [7]. 
The GAs differ from traditional search and optimization methods by four differences, they are:

- GAs search not a single point but a population of points in parallel,

- GAs do not require an auxiliary knowledge;

just the objective function and corresponding fitness levels influence thedirections of search.

- GAs, do not use deterministic ones but only probabilistic transition rules.

- GAs work on an encoding of the parameter set rather than the parameter set

itself (except in where real-valued individuals are used)[8].

\section{4-METHODOLOGY [9]}

In a genetic algorithm, a population of strings (called chromosomes or the genotype of the genome), which encode candidate solutions (called individuals, creatures, or phenotypes) to an optimization problem, is evolved toward better solutions. Traditionally, solutions are represented in binary as strings of $0 \mathrm{~s}$ and $1 \mathrm{~s}$, but other encodings are also possible. The evolution usually starts from a population of randomly generated individuals and happens in generations. In each generation, the fitness of every individual in the population is evaluated, multiple individuals are stochastically selected from the current population (based on their fitness), and modified (recombined and possibly randomly mutated) to form a new population. The new population is then used in the next iteration of the algorithm. Commonly, the algorithm terminates when either a maximum number of generations has been produced, or a satisfactory fitness level has been reached for the population. A typical genetic algorithm requires:

- a genetic representation of the solution domain,

- a fitness function to evaluate the solution domain.

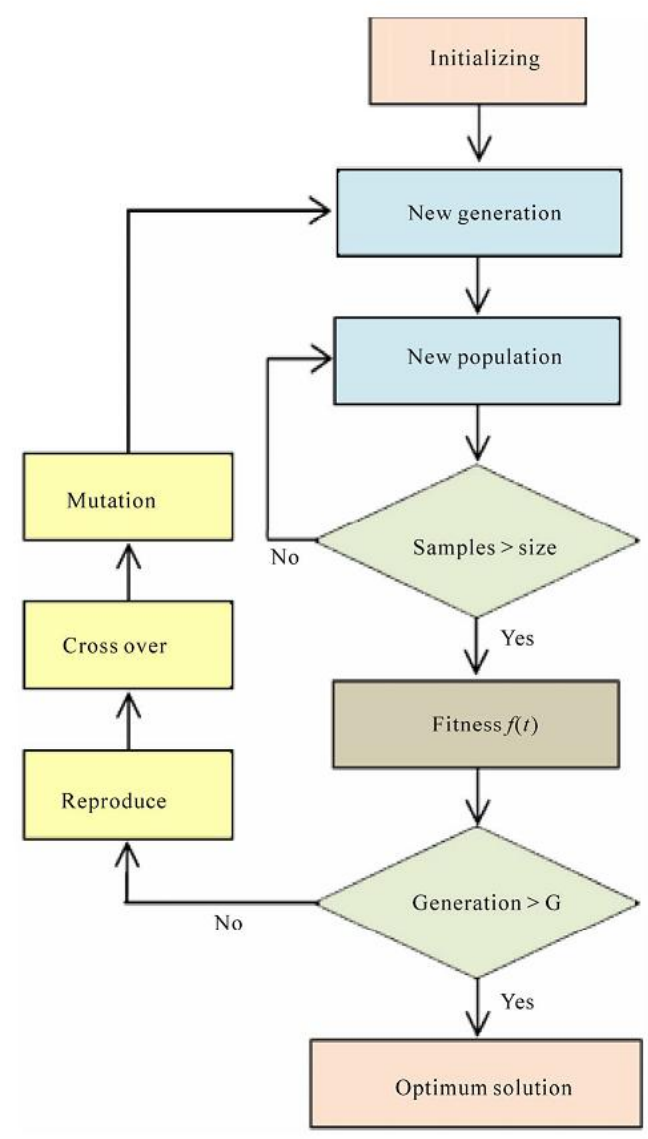

Fig.3 Genetic algorithm - program flow chart.

\section{5- SIMULATION RESULTS}

PID controller parameters will be optimized by applying GA. Here we use Matlab Genetic Algorithm to simulate it. The first and the most crucial step is to encoding the problem into suitable GA chromosomes and then construct the population. Each chromosome comprises of three parameters, $\mathrm{Kd}, \mathrm{Kp}$, Ki, with value bounds varied depend on objective functions used.

There are several variables used as the standard to measure systems performance. In general, unit step input is used to test the systems, and the output signals is characterized by some standard performance measures: delay time, rise time, percent overshoot, peak time, settling time, and error signal. All these measures are defined in time domain response.

Figure(4) below describes standard performance measures of a typical system driven by unit step input. The delay time is measured as the time needed by systems to reach from $50 \%$ of final value. There are several criteria for the rise time, actually in general, is measured as the time needed by systems to reach from 0 to $100 \%$ of final value or from $10 \%$ to $90 \%$ of final value. But, for measurement simplicity, we use $0-100 \%$ criterion. Percent overshoot is defined as the point where the system response reaches the peak. Peak time is the point 
where the maximum value reached (overshoot) at 3.2 second. settling time, for example $1 \%$ criterion, $2 \%$ criterion,

and $5 \%$ criterion. Here we use $5 \%$ criterion settling time. And error signal is the difference between the input signal magnitude and system response final magnitude. In this work four cases are taken :

The first case when $\Delta=0.75$ for deadzone (RAETFE) and $\zeta$ is variable from (0.1-1.0) as shown in table (1). While the second case when $\Delta=0.5$ for deadzone (RAETFE) and $\zeta$ is variable from (0.1-1.0) as shown in table (2).

The third case when $\zeta=0.3$ and $\Delta$ is variable from (0.1-1.0) as shown in table (3). While the fourth case when $\zeta=0.6$ and $\Delta$ is variable from (0.1-1.0) as shown in table (4)

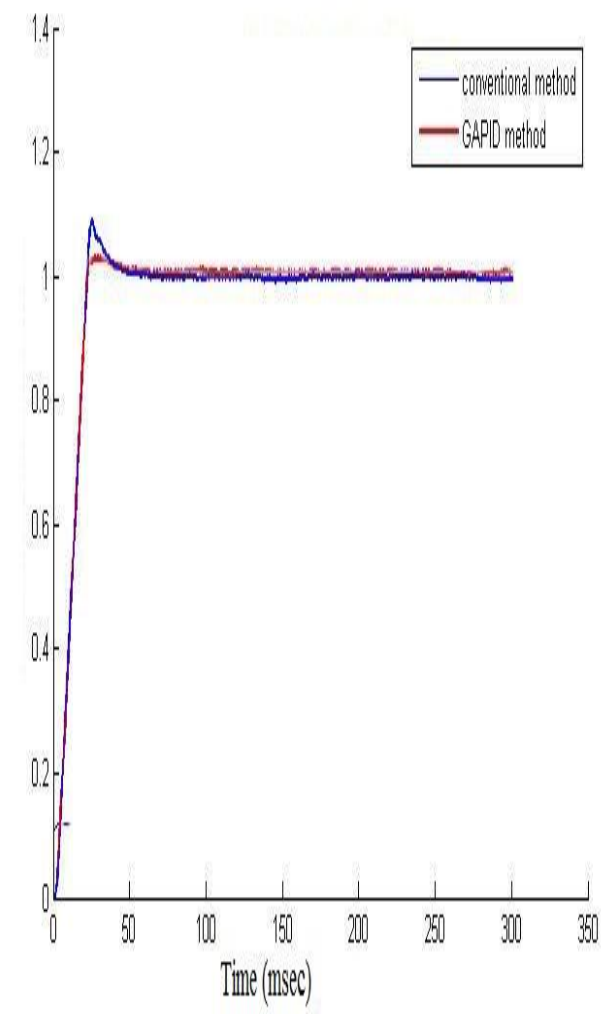

Fig.4 Standard performance measures of a typical system driven by unit step input for PID controller.

There are four tables for the four mentioned cases, which are in Table 1 shows the genetic algorithm optimum results for different gain values of PID and transient performance when $\Delta=0.75$ for dead zone (RAETFE) , ULIM $=200$ for saturation, $\Omega \mathrm{N}=144, \mathrm{HM}=3$ (HUGE MOMENT).
Table 1 Genetic Algorithm Optimum Results

\begin{tabular}{|c|c|c|c|c|c|c|c|c|}
\hline $\mathbf{Z}$ & $\mathbf{K p}$ & $\mathbf{K i}$ & $\mathbf{K d}$ & $\mathbf{M p}$ & $\begin{array}{c}\mathbf{T p} \\
(\mathbf{m s e c}\end{array}$ & $\begin{array}{c}\mathbf{T d} \\
(\mathbf{m s e c})\end{array}$ & $\begin{array}{l}\mathbf{T r} \\
(\mathbf{m s e c})\end{array}$ & $\begin{array}{c}\mathbf{T s} \\
(\mathbf{m s e c})\end{array}$ \\
\hline 0.1 & 754.5818 & 99.2441 & 75.3450 & 1.0132 & 38 & 12.241 & 29.35 & 180 \\
\hline 0.2 & 754.5818 & 99.2441 & 75.3450 & 1.0132 & 38 & 12.241 & 29.35 & 140 \\
\hline 0.3 & 677.6254 & 87.9769 & 67.3549 & 1.0132 & 38 & 12.241 & 29.35 & 140 \\
\hline 0.4 & 885.7227 & 90.0066 & 89.8315 & 1.02495 & 25 & 12.241 & 22.51 & 80 \\
\hline 0.5 & 915.9792 & 107.0014 & 93.1275 & 1.0132 & 38 & 12.241 & 29.35 & 140 \\
\hline 0.6 & 908.2300 & 116.6521 & 97.2499 & 1.0024 & 52 & 12.241 & 49.72 & 80 \\
\hline 0.7 & 951.0498 & 113.3742 & 98.5224 & 1.0132 & 38 & 12.241 & 29.35 & 140 \\
\hline 0.8 & 757.2700 & 101.2939 & 75.0834 & 1.0249 & 25 & 12.241 & 22.51 & 80 \\
\hline 0.9 & 733.3928 & 100.4799 & 76.4113 & 1.0132 & 38 & 12.241 & 29.35 & 140 \\
\hline 1.0 & 982.6898 & 91.4726 & 90.1229 & 1.0884 & 25 & 12.241 & 22.245 & 80 \\
\hline
\end{tabular}

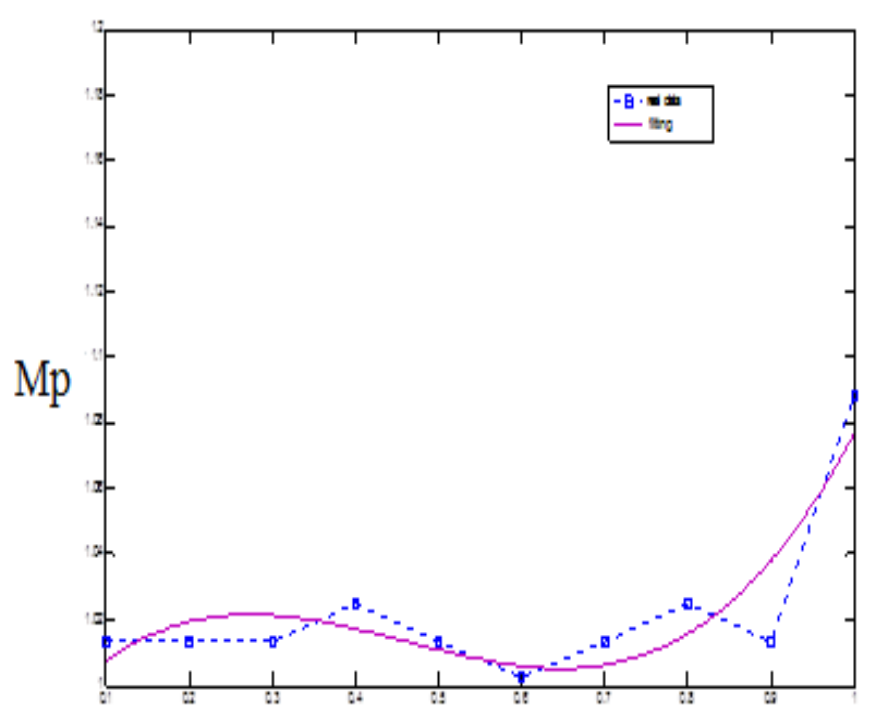

zeta

Fig.5 The relation between damping ratio zeta $\zeta$ max.peak overshoot $M_{p}$ when deadzone time delta $\Delta=0.75$ 


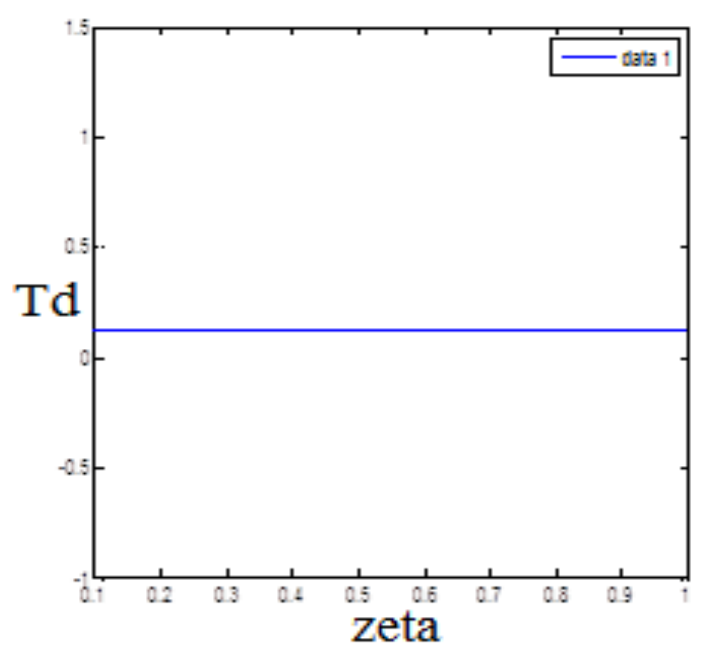

Fig.6 The relation between damping ratio zeta $\zeta \&$ delay time $T_{d}$ when deadzone time delta $\Delta=0.75$

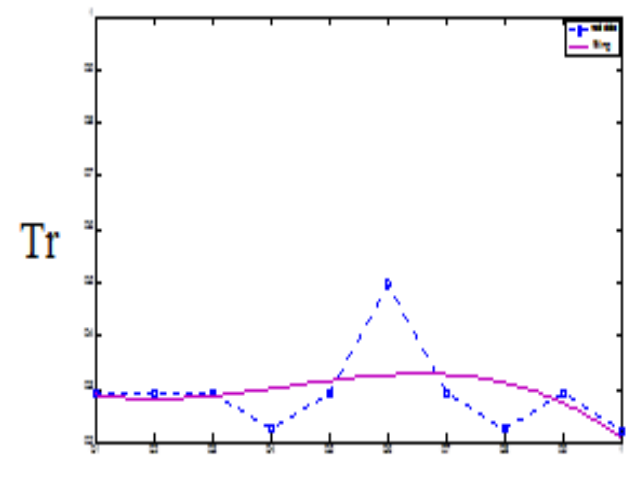

zeta

Fig.7 The relation between damping ratio zeta $\zeta \&$ rise time $T_{\mathrm{r}}$ when deadzone delta $\Delta=0.75$

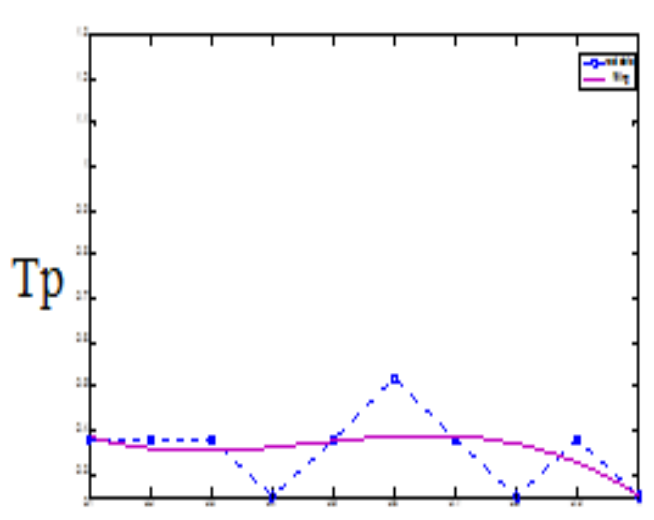

zeta

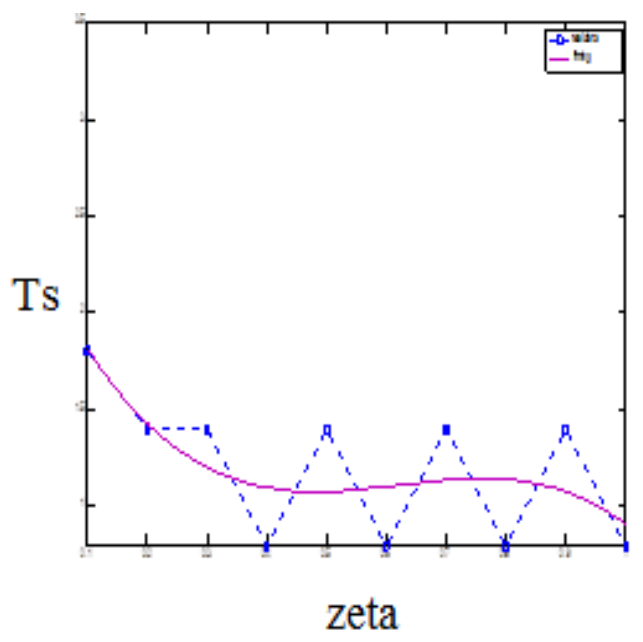

Fig.9 The relation between damping ratio zeta \& settling time $T_{s}$ when deadzone time delta $\Delta=0.75$

Table 2, shows genetic algorithm optimum results for different gain values of PID and transient performance when $\Delta=0.5$ for deadzone (raetfe), ulim $=200$ for saturation, $\omega n=$ $144, \mathrm{hm}=3$ (huge moment)

Table 2 Genetic Algorithm Optimum Results

\begin{tabular}{|c|c|c|c|c|c|c|c|c|}
\hline $\mathbf{Z}$ & $\mathbf{K p}$ & $\mathbf{K i}$ & $\mathbf{K d}$ & $\mathbf{M p}$ & $\begin{array}{c}\text { Tp } \\
(\mathbf{m s e c})\end{array}$ & $\begin{array}{c}\text { Td } \\
(\mathbf{m s e c})\end{array}$ & $\begin{array}{c}\text { Tr } \\
(\mathbf{m s e c})\end{array}$ & $\begin{array}{c}\text { Ts } \\
(\mathbf{m s e c})\end{array}$ \\
\hline 0.1 & 721.2435 & 75.6320 & 73.5623 & 1.0132 & 38 & 12.242 & 29.34 & 150 \\
\hline 0.2 & 637.2281 & 85.2942 & 65.5922 & 1.0132 & 38 & 12.242 & 29.35 & 150 \\
\hline 0.3 & 678.8693 & 96.6264 & 70.2872 & 1.0132 & 38 & 12.243 & 29.36 & 150 \\
\hline 0.4 & 707.4107 & 89.6187 & 73.3138 & 1.0132 & 38 & 12.242 & 29.36 & 140 \\
\hline 0.5 & 890.9687 & 95.4964 & 90.1083 & 1.0132 & 38 & 12.242 & 29.36 & 140 \\
\hline 0.6 & 970.9693 & 134.5488 & 101.8444. & 1.0032 & 45 & 12.242 & 37.69 & 85 \\
\hline 0.7 & 692.3050 & 108.5041 & 69.5329 & 1.0132 & 38 & 12.243 & 29.35 & 140 \\
\hline 0.8 & 950.2976 & 125.0130 & 94.0703 & 1.0249 & 25 & 12.242 & 22.51 & 90 \\
\hline 0.9 & 850.9007 & 124.6650 & 84.5597 & 1.0249 & 25 & 12.242 & 22.51 & 90 \\
\hline 1.0 & 846.0631 & 120.7570 & 87.8157 & 1.0132 & 38 & 12242 & 29.35 & 140 \\
\hline
\end{tabular}

Fig.8 The relation between damping ratio zeta $\&$ peak time $T_{p}$ when deadzone time delta $\Delta=0.75$ 


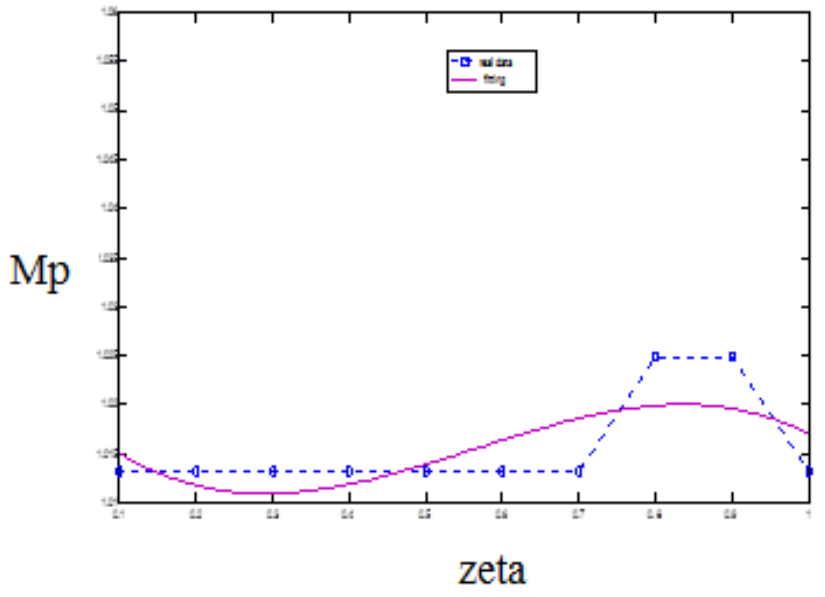

Fig.10 The relation between damping ratio zeta $\zeta$ \&max.peak overshoot $M_{p}$ when deadzone time delta $\Delta=0.5$

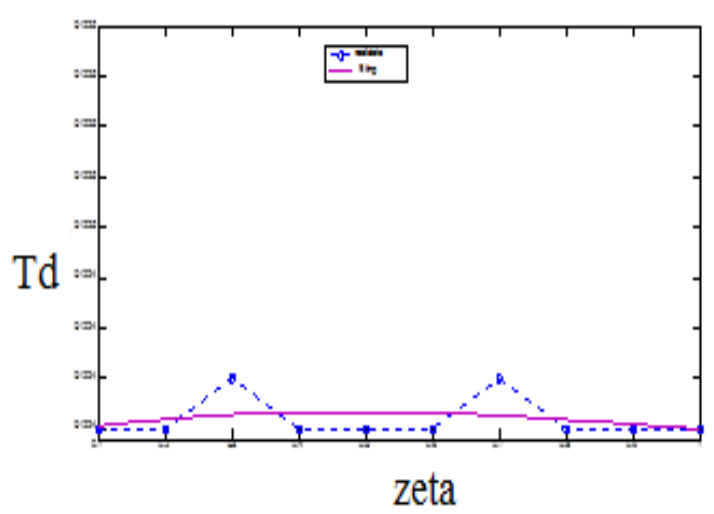

Fig.11 The relation between damping ratio zeta $\zeta \&$ delay time $T_{d}$ when deadzone time delta $\Delta=0.5$

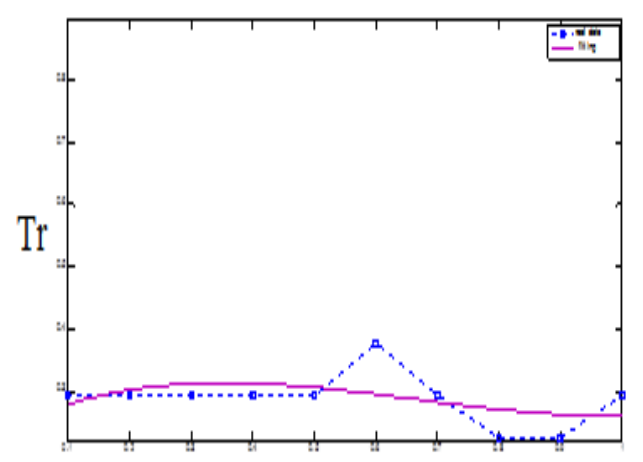

zeta

Fig.12 The relation between damping ratio zeta $\zeta \&$ rising time $T_{\mathrm{r}}$ when deadzone time delta $\Delta=0.5$

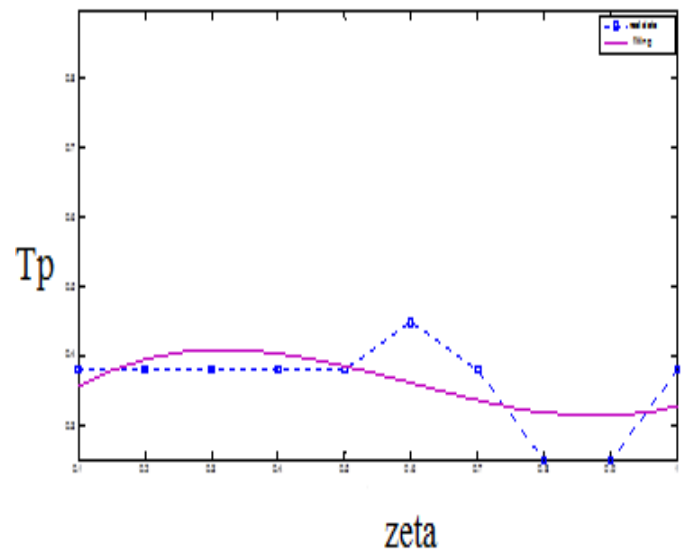

Fig.(13) The relation between damping ratio Zeta $\zeta \&$ Peak time $T_{p}$ when deadzone time delta $\Delta=0.5$

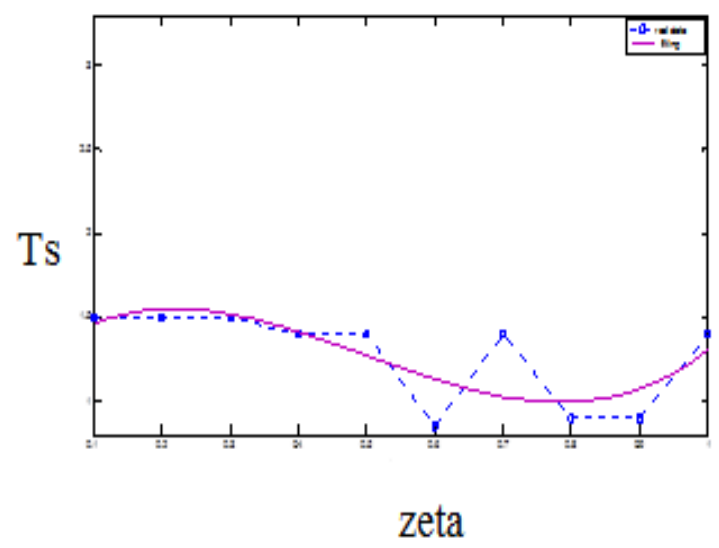

Fig.14 The relation between damping ratio zeta $\zeta \&$ settling time $T_{\mathrm{s}}$ when deadzone time delta $\Delta=0.5$

Table 3 shows the genetic algorithm optimum results for different gain values of pid and transient performance when $\zeta$ $=0.3$, ulim $=200$ for saturation, $\omega n=144, \mathrm{hm}=3$ (huge moment) 
Table 3 Genetic Algorithm Optimum Results

\begin{tabular}{|c|c|c|c|c|c|c|c|c|}
\hline$\Delta$ & Kp & Ki & Kd & Mp & $\begin{array}{c}\text { Tp } \\
(\mathbf{m s e c})\end{array}$ & $\begin{array}{c}\text { Td } \\
(\mathbf{m s e c})\end{array}$ & $\begin{array}{c}\text { Tr } \\
(\mathbf{m s e c})\end{array}$ & $\begin{array}{c}\text { Ts } \\
(\mathbf{m s e c})\end{array}$ \\
\hline 0.1 & 776.8372 & 106.0846 & 80.3730 & 1.0132 & 38 & 12.242 & 29.35 & 120 \\
\hline 0.2 & 785.783598 .7298 & 81.7990 & 1.0132 & 38 & 12.242 & 29.35 & 120 \\
\hline 0.3 & 616.9086 & 109.9305 & 61.6555 & 1.02965 & 31 & 12.222 & 22.51 & 80 \\
\hline 0.4 & 749.4216108 .4152 & 78.5293 & 1.0132 & 38 & 12.242 & 29.35 & 120 \\
\hline 0.5 & 812.1277121 .7033 & 84.3297 & 1.0132 & 38 & 12.241 & 29.35 & 120 \\
\hline 0.6 & 896.7755 & 163.9519 & 88.1837 & 1.02965 & 31 & 12.241 & 22.51 & 80 \\
\hline 0.7 & 925.1863 & 116.7138 & 98.7058 & 1.0024 & 52 & 12.241 & 49.7 & 80 \\
\hline 0.8 & 890.0490 & 221.5051 & 96.8994 & 1.0151 & 52 & 12.241 & 37.69 & 80 \\
\hline 0.9 & 962.1358 & 212.8717 & 101.4063 & 1.024 & 38 & 12.241 & 26.55 & 150 \\
\hline 1.0 & 912.1743 & 161.3187 & 93.8667 & 1.0132 & 38 & 12.241 & 28.93 & 120 \\
\hline
\end{tabular}

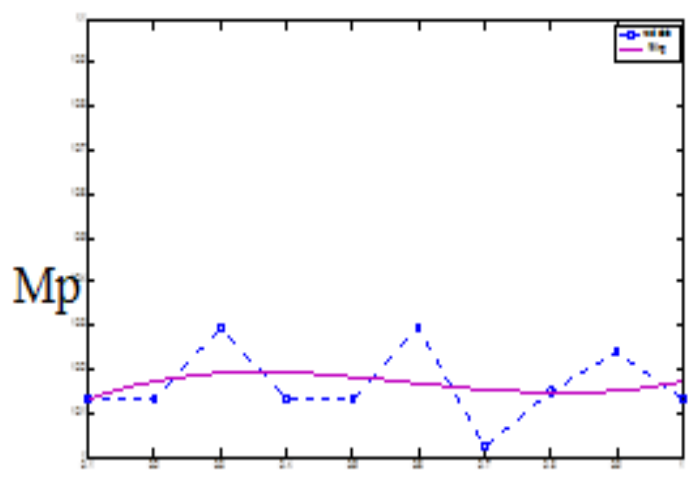

Delta

Fig.15 The relation between deadzone time delta $\Delta \&$ max. peak overshoot $\left(M_{p}\right)$ when damping ratio Zeta $\zeta=0.3$

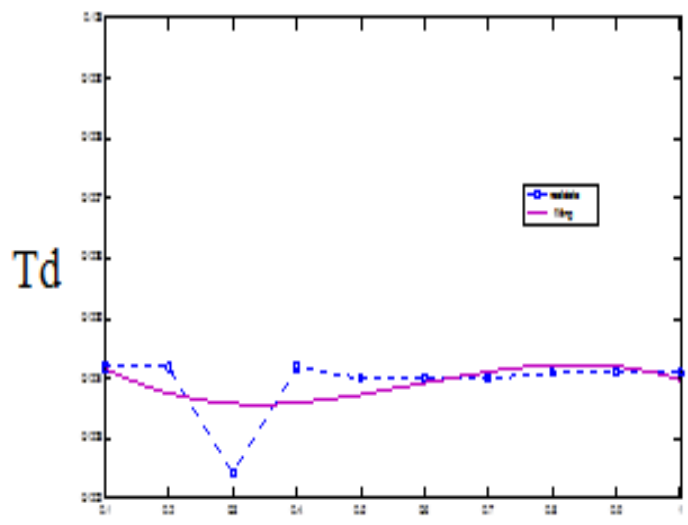

Delta

Fig.16 The relation between deadzone time delta $\Delta \&$ delay time $T_{d}$ when damping ratio zeta $\zeta=0.3$

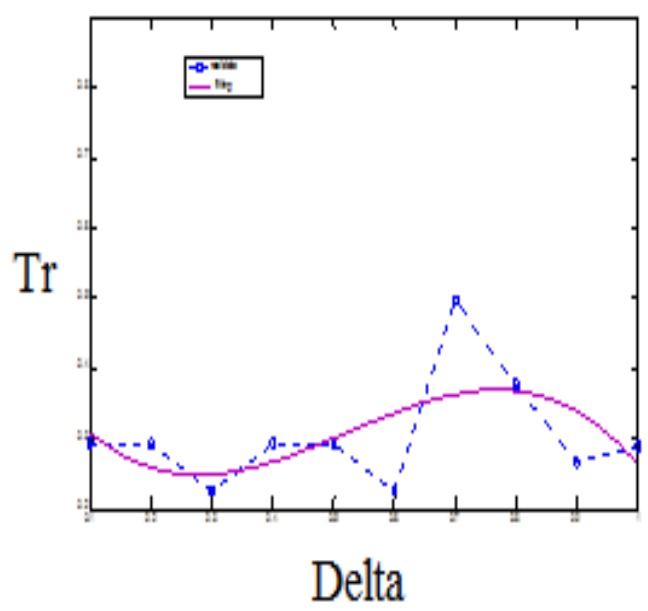

Fig.17 The relation between deadzone time delta $\triangle \&$ rising time $\left(T_{r}\right)$ when $\zeta=0.3$

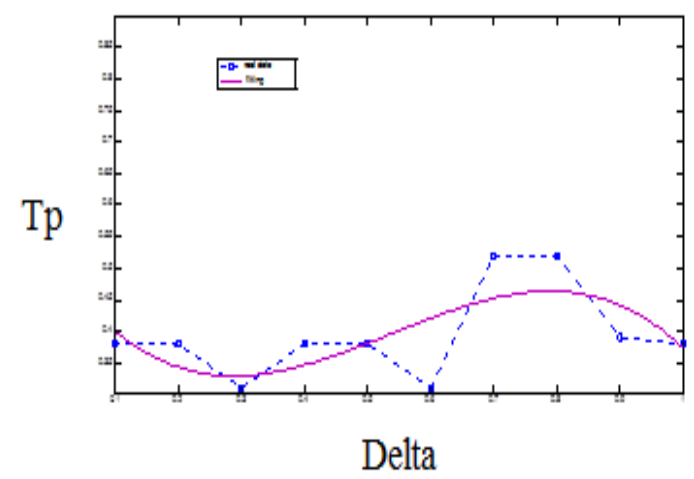

Fig.18 The relation between deadzone time delta $\Delta \&$ peak time $T_{p}$ when damping ratio zeta $\zeta=0.3$

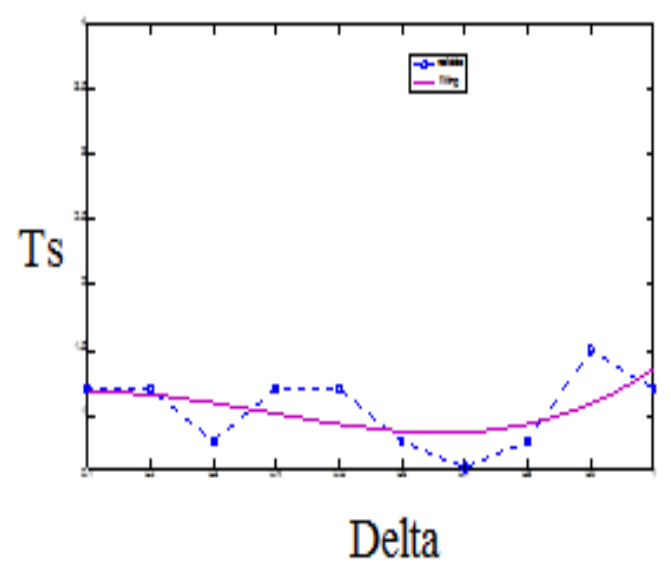

Fig.19 The relation between deadzone time delta $\Delta \&$ settling time $T_{\mathrm{s}}$ when damping ratio zeta $\zeta=0.3$

Table 4 shows the genetic algorithm optimum results for different gain values of pid and transient performance when $\zeta$ $=0.6$, ulim $=200$ for saturation, $\omega \mathrm{n}=144, \mathrm{hm}=3$ (huge moment). 


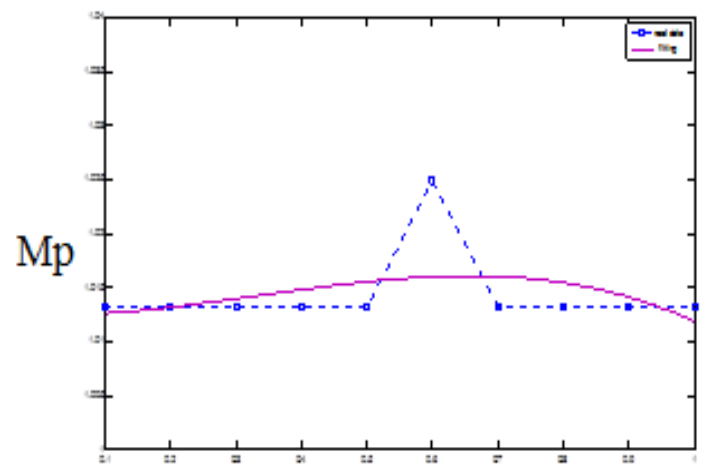

Delta

Fig.20 The relation between deadzone time delta $\Delta \&$ max. peak overshoot $\left(M_{p}\right)$ when $\zeta=0.6$

Table 4 Genetic Algorithm Optimum Results

\begin{tabular}{|c|c|c|c|c|c|c|c|c|}
\hline$\Delta$ & $\mathbf{K p}$ & $\mathbf{K i}$ & $\mathbf{k d}$ & $\mathbf{M p}$ & $\begin{array}{c}\text { Tp } \\
(\mathbf{m s e c})\end{array}$ & $\begin{array}{c}\text { Td } \\
(\mathbf{m s e c})\end{array}$ & $\begin{array}{c}\mathbf{T r} \\
(\mathbf{m s e c})\end{array}$ & $\begin{array}{c}\text { Ts } \\
(\mathbf{m s e c})\end{array}$ \\
\hline 0.1 & 626.7801 & 89.5452 & 64.4189 & 1.0132 & 38 & 12.245 & 29.36 & 160 \\
\hline 0.2 & 959.8850 & 176.5927 & 101.2775 & 1.0132 & 38 & 12.25 & 29.36 & 160 \\
\hline 0.3 & 857.4701 & 133.5264 & 86.0727 & 1.0132 & 38 & 12.245 & 29.36 & 170 \\
\hline 0.4 & 916.1705 & 98.1728 & 91.1001 & 1.0132 & 38 & 12.245 & 29.36 & 170 \\
\hline 0.5 & 949.8885 & 156.1254 & 96.3753 & 1.0132 & 38 & 12.242 & 29.36 & 170 \\
\hline 0.6 & 695.2232 & 72.945 & 68.8030 & 1.0249 & 25 & 12.242 & 22.51 & 125 \\
\hline 0.7 & 727.0700 & 85.9769 & 75.8768 & 1.0132 & 38 & 12.242 & 29.36 & 160 \\
\hline 0.8 & 973.6226 & 129.6789 & 97.6081 & 1.0132 & 38 & 12.245 & 29.36 & 170 \\
\hline 0.9 & 807.6227 & 83.1211 & 81.8213 & 1.0132 & 38 & 12.245 & 29.36 & 170 \\
\hline 1.0 & 894.7265 & 126.0383 & 92.6512 & 1.0132 & 38 & 12.245 & 29.36 & 170 \\
\hline
\end{tabular}

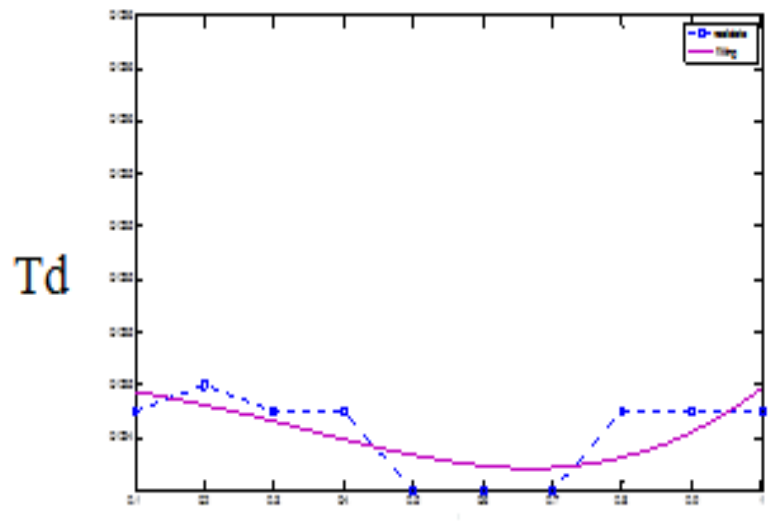

Delta

Fig.21 The relation between between deadzone time delta $\Delta \&$ delay time $\left(\mathbf{T}_{\mathrm{d})}\right.$ when $\zeta=0.6$

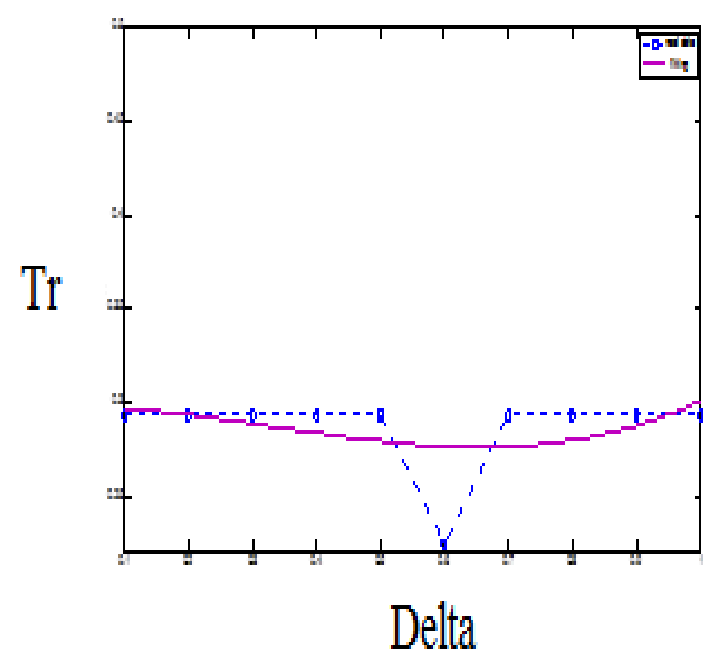

Fig.22 The relation between between deadzone time delta $\Delta$ \&rRising time $\left(T_{r}\right)$ when $\zeta=0.6$

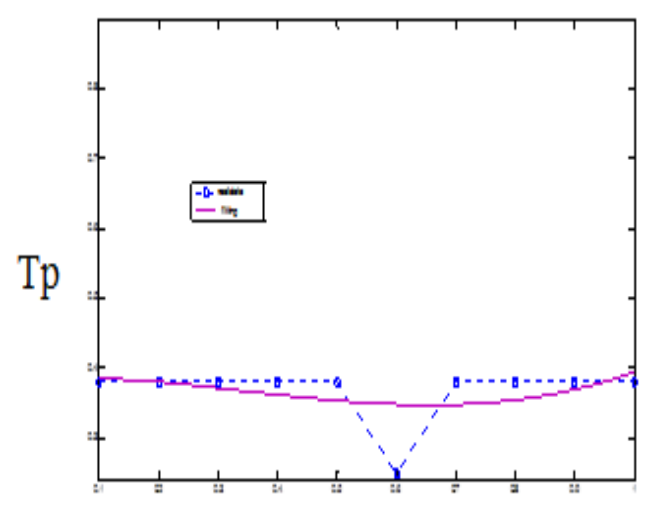

Delta

Fig.23 The relation between between deadzone time delta $\Delta \&$ peak time $\left(T_{p}\right)$ when $\zeta=0.6$ 


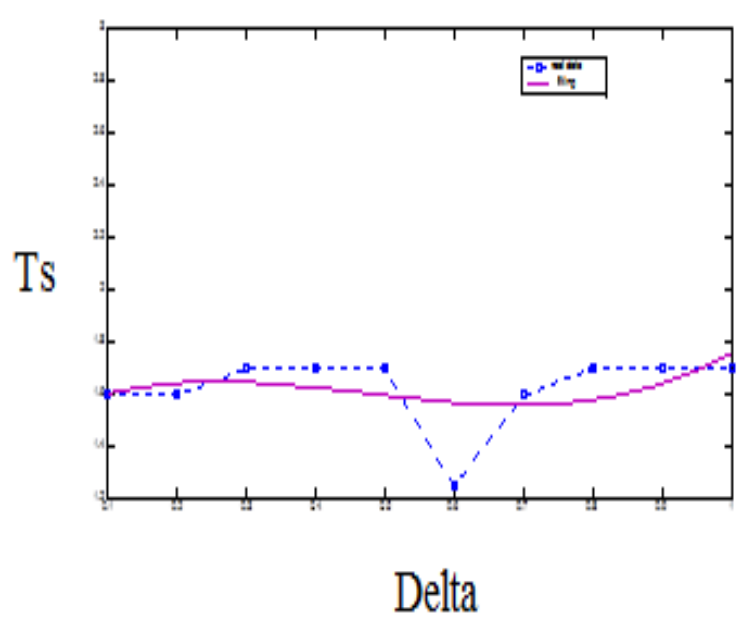

Fig.24 The relation between between deadzone time delta $\Delta \&$ sittling time $\left(T_{\mathrm{s})}\right.$ when $\zeta=0.6$

\section{6- CONCULUSION}

- $\quad$ The genetic algorithm based PID tuning provides much better results compared to the conventional methods as shown in figure (4).

- The conventional method is good for getting the initial values of the PID tuning which needs to be modified.

- In the designed PID controller tuning with GA, the actual response was found to be satisfying the required value. PID controller gain values depend upon the range selected for the initial population. The range of requirement can be widened by increasing the range of initial population but the number of generations required to converge to optimal value may increase.

- Genetic Algorithm applied in PID controller improves transient response. This is shown by average percent overshoot reduction about $80 \%$ compared to the conventional methods such as genetic PID optimization and genetic algorithm optimized fuzzy supervisory PID, while keep the delay time, rise time and peak time almost unchanged and improves the settling time as shown in figures (6) to figure (24).

\section{7- REFERENCES}

[1] Milan R. Ristanovic, Dragan V. Lazic and Ivica Indin (2008), "Nonlinear PID Controller Modification of The Electromechanical Actuator System for Aerofin Control with a PWM Controlled D.C. Motor", Automatic Control and Robotics Vol. 7, No.1, 2008 pp.131-139.

[2] Bindu R., Mini K. Namboothiripad (2012) "Tuning of PID Controller for DC Servo Motor using Genetic Algorithm" International Journal of Emerging Technology and Advanced Engineering Volume 2, Issue 3, pp.310-317March 2012.

[3] Victor M. Becerra, (2009), Nonlinear Control, Lecture 8, University of Reading, School of Systems Engineering.

[4] Mohamed Farid Bin Mohamed Faruq(2008),"PID Controller Design For Controlling DC Motor Speed
Using MATLAP Application". Universiti Malaysia Pahang, Electrical \& Electronics Engineering.

[5] Chung-Hee Yoo, Young-Cheol Lee and Sang-Yeal Lee (2005)," A Robust Controller for an Electro-Mechanical Fin Actuator", Agency for Defense Development, South Korea.

[6] Shaimaa A. Mahdi, Zeina A. Abdul Redha (2011) "The Linear and Non-linear electromechanical Fin actuators" Al-Khwarizmi Engineering Journal Vol.7 , No. 4 , 2011 pp.88-96.

[7] Ayman A. Aly(2011)," PID Parameters Optimization Using Genetic Algorithm Technique for Electrohydraulic Servo Control System" Intelligent Control and Automation Journal Vol.2 , 2011 pp. 69-76.

[8] Andri Mirzal, Shinichiro Yoshii, Masashi Furukawa (2003)"PID Parameters Optimization by Using Genetic Algorithm" Graduate School of Information Science and Technology Hokkaido University Sapporo, Japan.

[9] Nibal Fadel Farman(2013), "Performance of the Solar Adsorption Refrigeration System under Various Conditions and Parameters for Iraq Climate " A thesis Submitted to the Energy Engineering Department, College of Engineering, University of Baghdad in Partial Fulfillment of the Requirements for the Degree of Doctor of Philosophy in Energy Engineering. 


\section{8- NAMENCLATUR}

\begin{tabular}{||c||l||l||}
\hline Symbol & \multicolumn{1}{|c||}{ Meaning } & Unit \\
\hline \hline$\xi$ & Damping ratio & \\
\hline$\omega n$ & Natural Frequency & Rad /sec \\
\hline $\mathrm{Kp}$ & Proportional gain & \\
\hline $\mathrm{Ki}$ & Integral gain & \\
\hline $\mathrm{Kd}$ & Derivative gain & \\
\hline$\Delta$ & Deadzone delay time & \\
\hline $\mathrm{Mp}$ & Maximum peak overshoot & \\
\hline $\mathrm{Td}$ & Delay time & Sec. \\
\hline $\mathrm{Tr}$ & Rising time & Sec. \\
\hline $\mathrm{Tp}$ & Peak time & Sec. \\
\hline $\mathrm{ts}$ & Settling time & Sec. \\
\hline
\end{tabular}

